livraisons

d'Histoire

de l'Architecture

\section{Livraisons de l'histoire de l'architecture}

$30 \mid 2015$

Le dessin d'architecture : œuvre/outil des architectes

?

\title{
Fantômes et revenants : les dessins français d'architecture gothique
}

Ghosts and revenants: French gothic architectural drawings

Geister und Wiederauferstandene: gotische Architektur in französischen

Zeichnungen

Étienne Hamon

\section{CpenEdition}

Journals

Édition électronique

URL : http://journals.openedition.org/lha/572

DOI : $10.4000 /$ lha. 572

ISSN : 1960-5994

Éditeur

Association Livraisons d'histoire de l'architecture - LHA

Édition imprimée

Date de publication : 30 décembre 2015

Pagination : 13-27

ISSN : $1627-4970$

Référence électronique

Étienne Hamon, «Fantômes et revenants : les dessins français d'architecture gothique », Livraisons de I'histoire de l'architecture [En ligne], 30 | 2015, mis en ligne le 18 décembre 2017, consulté le 06 juin 2020. URL : http://journals.openedition.org//ha/572 ; DOI : https://doi.org/10.4000//ha.572 
Par Étienne HAMON

\section{FANTÔMES ET REVENANTS : LES DESSINS FRANÇAIS D’ARCHITECTURE GOTHIQUE}

S'il est un domaine de l'histoire de l'art gothique où la France accuse, eu égard à la qualité de son patrimoine, un retard criant sur ses voisins, c'est bien celui du dessin d'architecture. Le terrain est, avouons-le, semé d'embûches. Redécouvert il y a deux siècles en même temps que l'archéologie médiévale, le dessin d'architecture de cette période reste avant tout un objet de fantasmes. Bien au-delà du monde de l'archéologie, il fascine en effet par son étrangeté, son ancienneté et sa rareté. La bibliographie est à la mesure de cette séduction : volumineuse, concentrée sur une poignée de documents insatiablement revisités, et souvent hautement spéculative quand il s'agit, par exemple, de reconstituer les tracés directeurs de dessins dont on sait le support - le parchemin en général - supérieurement résistant mais instable.

Or le renouveau des études sur l'art français de la fin de l'époque gothique, qu'elles portent sur le processus de création architectural ou sur la culture technique et visuelle de ses acteurs, se heurte aujourd'hui à la pénurie de corpus raisonné de ces dessins. Car malgré des avancées récentes dans la connaissance de l'objet et l'exemple donné par quelques publications de corpus régionaux ou nationaux, les pièces issues du domaine français attendent toujours un recensement et des notices approfondies assortis d'études thématiques mobilisant, dans le même élan, les références aux pièces disparues. Le moment est opportun pour y remédier car les perspectives n'ont jamais été aussi prometteuses. Depuis qu'archivistes, historiens et historiens de l'art ont réinvesti le champ des arts graphiques et des sources documentaires de la création, il y a une trentaine d'années, les découvertes de nouveaux dessins se sont multipliées, tandis que la numérisation des fonds iconographiques et leur mise en ligne laissent, depuis peu, entrevoir une nouvelle moisson.

En attendant, on est condamné à raisonner sur une base de quelques dizaines de pièces connues qui forment, pour la France entre le XIII ${ }^{e}$ siècle et le $\mathrm{XVI}^{\mathrm{e}}$ siècle, un ensemble disparate d'un point de vue géographique, chronologique, technique et plastique, alors que les œuvres construites alors selon les principes de la projection graphique se comptent encore par milliers. Il est donc trop tôt pour proposer du phénomène une approche globale renouvelée. Il n'est pas inutile, en revanche, de revenir sur certains aspects de son historiographie. Chaque pièce étant unique matériellement, c'est d'abord la fortune ou plutôt l'infortune que ces outils de médiation si singuliers ont connu à l'époque moderne qui les distingue dans l'histoire de l'archéologie médiévale. 


\section{Enjeux}

Quelle qu'en soit la qualité, l'immense littérature sur le dessin d'architecture gothique rend compte, consciemment ou non de la part de ses auteurs, de l'importance de ce support dans la compréhension du processus de création. L'époque gothique marque un tournant dans l'histoire du dessin d'architecture. Elle en constitue le berceau, du moins pour la résurgence des formes techniquement les plus élaborées, que l'on se fie aux témoignages des textes ou aux œuvres conservées. En tant que tel ou à travers les sources qui y renvoient, il est donc un matériau de premier ordre pour la connaissance de l'architecture, des architectes et des modes de représentation du bâti à la fin du Moyen Âge, sans parler des questions de médiation et de transmission des modèles, à condition de ne pas se méprendre sur ses codes et sur son statut. L'étude du dessin d'architecture médiéval appelle donc un va et vient critique entre les trois principaux témoins du processus de création, lesquels se répondent rarement sur le terrain mais s'éclairent toujours mutuellement même en l'absence de liens historiques qui les unissent: les outils graphiques, les textes et les monuments.

Les textes et les mentions indirectes au nombre desquelles on peut ranger le Carnet de Villard de Honnecourt ${ }^{1}$ nous apprennent que le dessin à l'échelle était en usage sur les grands chantiers du début du XIII ${ }^{e}$ siècle sous forme de plans, coupes et élévations géométrales, que passé le milieu de ce siècle il était omniprésent dans le processus de création artistique, au sens large (le marché pour la châsse de Nivelle y renvoie explicitement en 1272), et qu'il se perfectionna pour revêtir, dans les années 1300 , presque tous les aspects et toutes les fonctions qu'on lui connaît à l'époque moderne. Ils nous assurent, enfin que, dès le XIV e siècle, le dessin d'architecture pouvait être regardé comme une œuvre d'art à part entière, tandis que l'on en reconnaissait la propriété intellectuelle à son auteur. À ces caractéristiques, l'époque moderne apporta peu de bouleversements, le support le plus répandu depuis l'antiquité, le parchemin, restant en usage en plein XVII ${ }^{e}$ siècle. Les avancées touchèrent à la normalisation des systèmes scalaires et des codes d'usage des lavis ; à de nouvelles conventions pour la représentation du volume ; à la généralisation des maquettes en volume, dont l'emploi dans la France gothique reste encore l'objet de débats.

\section{Un corpus résiduel mais exemplaire}

Épures mises à part, les épaves, en France, de l'activité graphique des bâtisseurs gothiques, qu'il s'agisse des commanditaires, des architectes ou des peintres employés par les uns ou les autres, suffisent à illustrer les différentes étapes de l'évolution

1. Sa dernière édition en date est celle de Carl F. Barnes: The portfolio of Villard de Honnecourt, Paris, Bibliothèque nationale de France, MS Fr 1909: a new critical edition and color facsimile, Farnham, Burlington, Ashgate, 2009. 


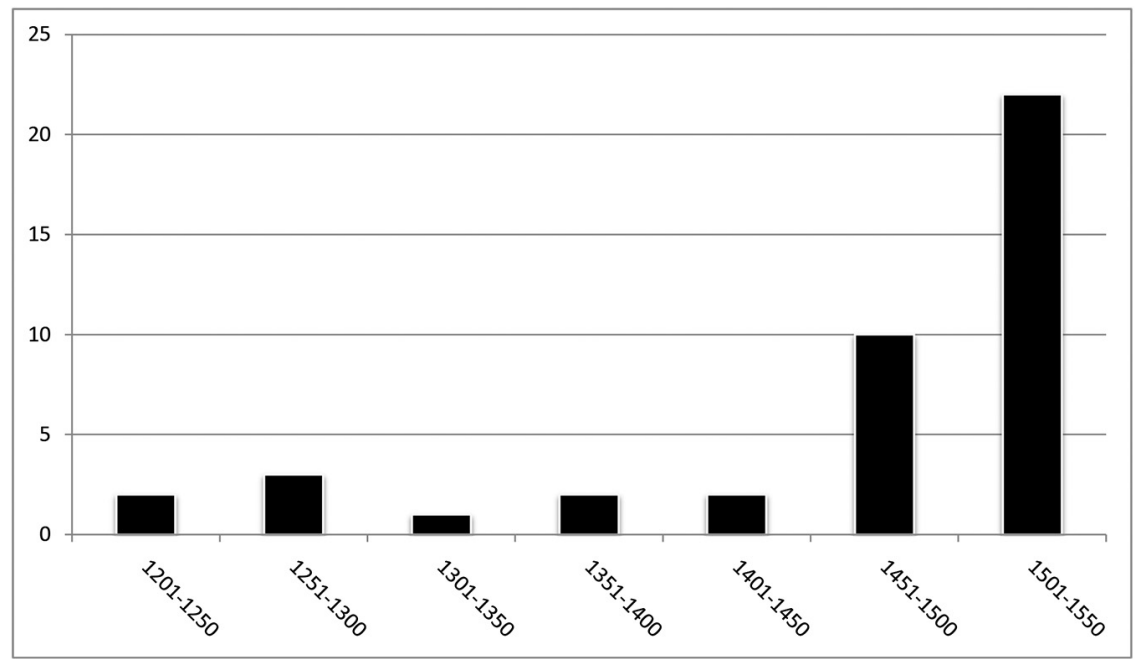

Ill. 1 : Nombre de dessins d'architecture gothiques français conservés par période. (C) Cliché Étienne Hamon.

du dessin et la diversité de ses formes et fonctions. Les plus anciens témoins originaux remontent au milieu du XIII ${ }^{\mathrm{e}}$ siècle, mais l'essentiel appartient aux $\mathrm{XV}^{\mathrm{e}}$ et $\mathrm{XVI}^{\mathrm{e}}$ siècles (ill. 1). Ils renvoient, selon les cas, à des usages dans la conception, la production, la diffusion, ou la réception. Et ils expriment des finalités techniques, médiatiques et, dans une moindre mesure chez nous, didactiques.

À côté de séries étrangères riches pour certaines de centaines de pièces et bénéficiant d'éditions récentes ${ }^{2}$, la France, berceau de l'art gothique et dont le patrimoine en la matière est le plus riche au monde, fait pâle figure avec ses quelques dizaines de dessins en attente de récolement et, à quelques exceptions près, rarement convoqués dans les essais sur l'histoire du dessin d'architecture. Les raisons de ce double décalage sont nombreuses. Elles tiennent à l'histoire des institutions, au statut incertain de ces documents au regard des cadres de classement des archives, à la dispersion précoce des collections thématiques nationales et à un certain désamour de la communauté scientifique jusqu'à une date récente.

Ainsi l'espace capétien n'a jamais abrité d'institutions de conservation des archives de chantier aussi émancipées et structurées que les fabriques des grandes églises des cités italiennes ou allemandes (Florence, Milan, Strasbourg, Cologne...). Ceux des dessins d'architecture qui n'étaient pas restés dans les mains de leurs auteurs trouvèrent difficilement place dans les cadres de classement des archives des commu-

2. Pour le monde germanique, il faut désormais compter avec les recueils de Hans-Joseph Böker commencés en 2005 avec les 425 dessins de Vienne (Architektur der Gotik. Bestandskatalog der weltgrössten Sammlung an gotischen Baurissen (Legat Franz Jäger) im Kupferstichkabinett der Akademie der bildenden Künste Wien, Salzburg, 2005) ; pour l'Italie du XIV siècle, la référence est désormais Valerio Ascani, Il Trecento disegnato. Le basi progettuali del'architettura gotica in Italia, Rome, 1997. 
nautés ou des administrations élaborés sous l'Ancien régime et normalisés au cours du XIX siècle. Le statut des documents figurés produits en même temps que les documents écrits y a longtemps été subalterne en raison de leur faible valeur économique ou juridique. À l'époque moderne, la France a vu s'exiler ses collections privées de dessins d'architecture telles que celles qui, aujourd'hui, forment le fonds de l'Académie de Vienne. Enfin par leurs caractéristiques matérielles, à commencer par l'irrégularité de leur format, les dessins de commande furent les principales victimes de l'ouverture de séries graphiques factices dans les dépôts d'archives.

Le mal - réemplois, dispersions, soustractions - était fait quand, vers le milieu du XIX ${ }^{\mathrm{e}}$ siècle, archéologues et archivistes prirent conscience de l'intérêt de ces dessins, à défaut de toujours respecter l'unité des fonds garante de leur intelligibilité. En 1878, le Musée des Archives départementales, florilège des plus beaux documents conservés dans les dépôts départementaux, rassemblé à l'occasion de l'Exposition universelle, ne recensait que deux documents de cette nature, tous deux miraculeusement préservés dans leur fonds d'origine du fait que le dessin était tracé sur le même support que le contrat qu'il illustrait ${ }^{3}$. Or la grande majorité des dessins français rescapés n'offre pas ces caractéristiques. Leur conservation actuelle est donc soit le fruit d'un salutaire désintérêt pour leur série d'origine, soit au contraire l'aboutissement d'un parcours chaotique jalonné de remplois, d'oublis, de disparitions et de réapparitions.

\section{Une érudition à éclipses}

L'histoire du dessin d'architecture médiéval connut un tournant vers 1840 quand apparurent les premières éditions scientifiques. L'attention se focalisa alors sur des codex du XIII ${ }^{\mathrm{e}}$ siècle entrés depuis peu dans des dépôts publics, à commencer par le Carnet de Villard de Honnecourt de la Bibliothèque royale. Sans rouvrir les débats sur sa fonction et sur le profil de son auteur, on rappellera qu'il suivit un parcours pour le moins sinueux : peut-être encore en usage comme carnet de modèles monumentaux à Paris vers 1430, il semble être entré en possession de la famille Félibien à la fin du XVI ${ }^{\mathrm{e}}$ siècle et au plus tard en 1666, avant d'échoir dans la bibliothèque de Saint-Germain-des-Prés où les révolutionnaires le saisirent ${ }^{4}$. Ses superbes dessins furent en partie gravés quelques années plus tard par Nicolas-Xavier Willemin dans ses Monuments français inédits (t. I, pl. 102 et suiv.). Cette collection entreprise en 1806 et publiée de façon posthume en 1839 perpétuait une tradition illustrée par Gaignières : les préoccupations de son éditeur allaient d'abord aux costumes des personnages représentés. Cette publication révéla l'existence du recueil aux pionniers

3. Musée des archives départementales. Recueil de fac-similés héliographiques de documents tirés des archives des préfectures, mairies et hospices. Par Gustave Adolphe Desjardins, Paris, Imprimerie nationale, 1878, $\mathrm{n}^{\circ} 137$ et pl. LII (projet de portail pour Saint-Jacques-aux-Pèlerins), no 140 et pl. LI (projet de châsse pour la cathédrale de Noyon).

4. Étienne Hamon, "Une source insoupçonnée de l'architecture flamboyante parisienne: le Carnet de Villard de Honnecourt ", Bulletin monumental, 2007, p. 281-288. 
de l'archéologie. Quicherat en donna en 1849 une notice illustrée dans la Revue archéologique; Lassus en prépara une édition qui parut en 1858, après sa mort, en même temps que celle de Willis à Londres (1859). Entre-temps, en 1846, Didron avait publié un autre recueil de dessins connu depuis sous le nom de Palimpseste de Reims (Arch. dép. Marne, G 661), obituaire dans lequel furent remployées, après grattage et recoupage, plusieurs peaux couvertes de dessins à la finalité toujours discutée 5 .

Archéologues ou architectes, ces savants n'hésitèrent pas à mobiliser leurs réseaux dans le monde du clergé ou des antiquaires pour se constituer leurs propres collections de documents anciens. Ainsi le grand dessin du jubé du Mans aujourd'hui conservé dans les collections du musée municipal fut-il d'abord "récupéré " par Lassus à la faveur de ses travaux dans la cathédrale à partir de 1848. L'architecte avait sans doute prévu de le publier, ce que fit son complice Hucher des années plus tard ${ }^{6}$. Plus tôt, Alexandre Lenoir avait transmis à son fils Albert un dessin trouvé dans les archives du château de Gaillon qu'Achille Deville, qui rapporte la chose, publia en 1850 en le mettant en rapport avec les travaux du cardinal d'Amboise des années 1500, sans égards pour son archaïsme formel. Il n'est plus localisable aujourd'hui (ill. 2) ${ }^{7}$. En ce milieu du XIX ${ }^{\mathrm{e}}$ siècle, les sociétés savantes jouèrent un rôle déterminant dans la redécouverte de ces dessins, comme on le verra encore plus loin dans le cas de la Normandie. La Société archéologique de Montpellier publia ainsi dans ses Mémoires, en 1850, un dessin de portail alors en mains privées, qui tomba ensuite dans l'oubli pour un siècle (ill. 3).

D'abord «Monument » ou objet de collection, le dessin d'architecture médiéval accéda tardivement au statut d'objet d'étude en tant que tel. Il y parvint indirectement en endossant le rôle de support pédagogique pour l'enseignement de l'archéologie et de la diplomatique. En 1887, la Société de l'École des chartes sélectionna ainsi quelques planches du Carnet de Villard dans son "Album paléographique ". Mais un premier fac-similé de dessin gothique était entré dans les collections de l'École quelques années auparavant : celui du portail de Saint-Jacques-aux-Pèlerins à Paris (1473), qui, à une époque pas si éloignée de la nôtre, fut retiré des grands registres à onglets des chartes de l'hôpital pour être mis sous cadre dans le bureau du conservateur des archives de l'AP-HP. Avec un autre projet dessiné pour la châsse de la cathédrale de Noyon (1499), il avait été sélectionné par Brièle parmi les 170 documents du Musée des archives départementales ( $\mathrm{n}^{\circ} 137$ et pl. LII) pour

5. Adolphe Napoléon Didron, "Dessins palimpsestes du XIII" siècle ", Annales archéologiques, t. V, 1846, p. 87-94.

6. Eugène Hucher, "Le jubé du cardinal Philippe de Luxembourg à la cathédrale du Mans », Bulletin de la société d'agriculture, Sciences et arts de la Sarthe, t. 12, 1869-1870, p. 321-365. E. Hucher, Le Jubé du cardinal Philippe de Luxembourg à la cathédrale du Mans : décrit d'après un dessin d'architecte du temps et des documents inédits, Le Mans, 1875.

7. Achille Deville, Comptes et dépenses de la construction du château de Gaillon, Paris, 1850, p. CVIICVIII et pl. XIV. À supposer qu'il soit authentique, le dessin montre des graphismes caractéristiques des années 1400 incompatibles avec le décor de la chapelle de Gaillon et une attribution à Colin Biart, vers 1500, comme l'imaginait Deville. 


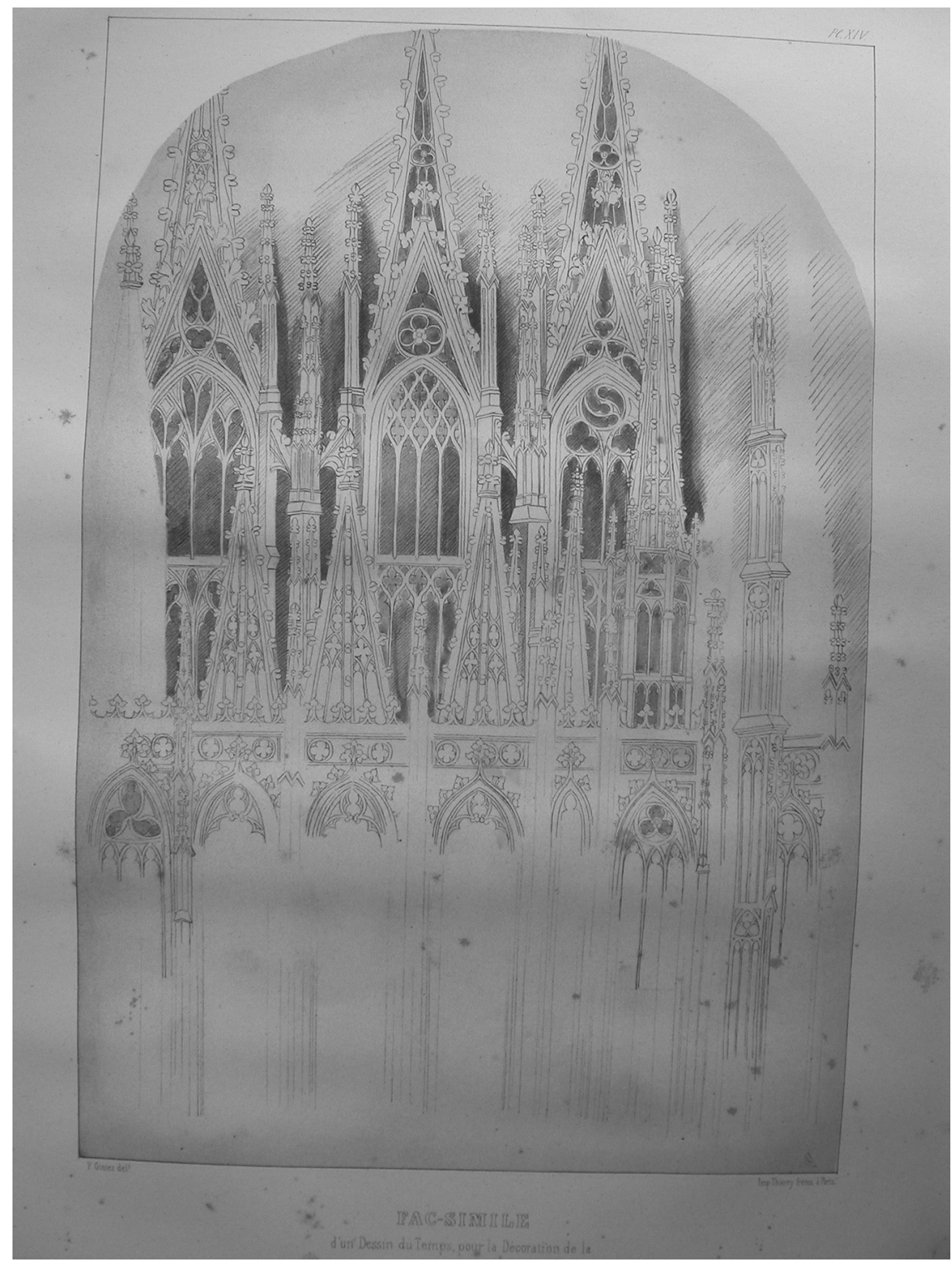

Ill. 2 : Fac-similé d'un dessin du temps pour la décoration de la chapelle du château de Gaillon. D’après A. Deville, Comptes et dépenses..., op. cit.

constituer le recueil publié par la Commission des archives départementales du Ministère de l'intérieur à l'occasion de l'Exposition universelle de 1878. La publication était supervisée par Jules Quicherat, alors directeur de l'École, et Léopold Delisle. Sa reproduction bénéficia du nouveau procédé d'héliogravure perfectionné 


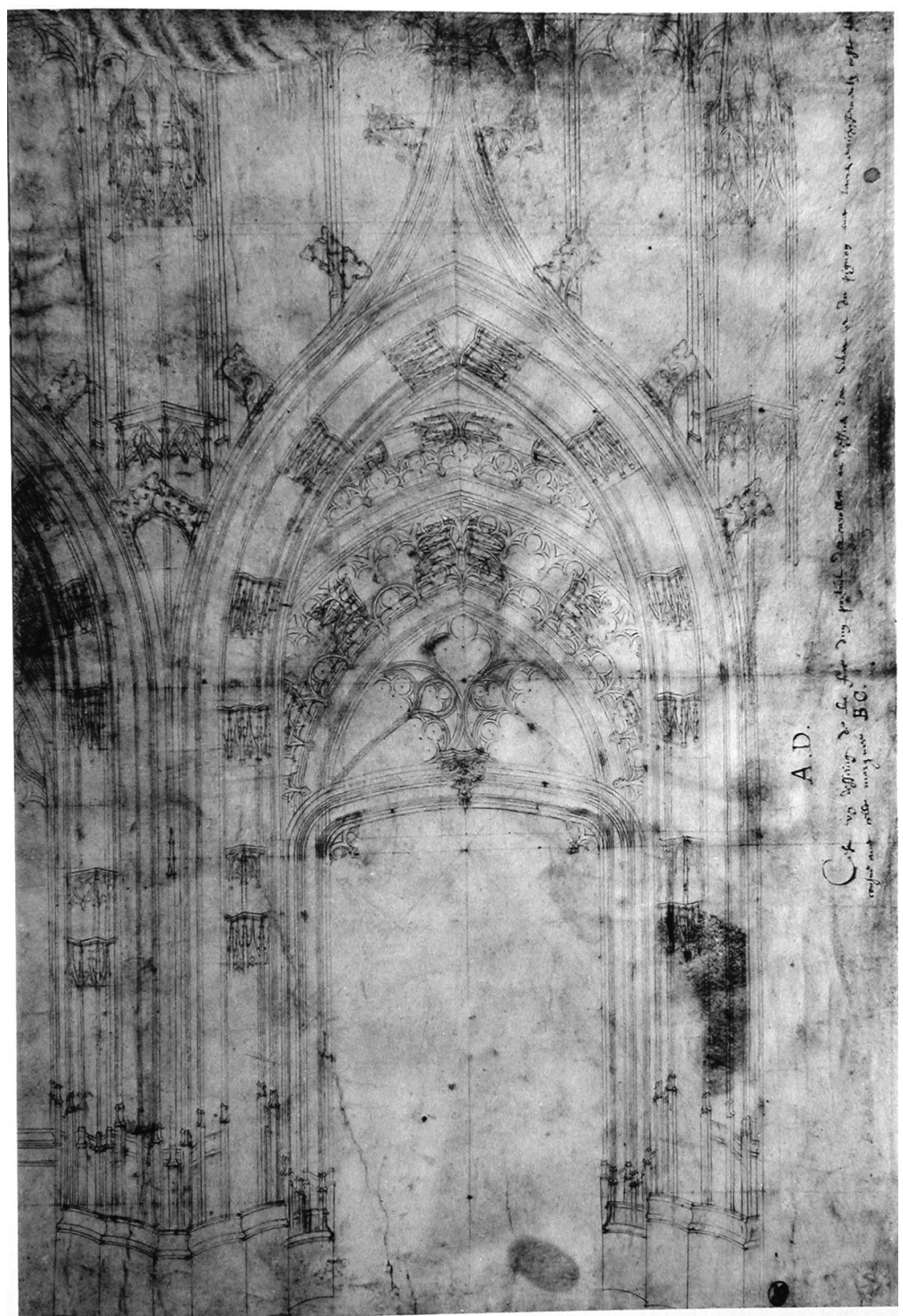

Ill. 3 : Projet de portail, fin du XV siècle. New York, Cloisters museum, accession number 68.49. Encre sur parchemin. D’après R. Branner, "A Fifteenth-century... ", op. cit. 
par Dujardin, employé depuis 1872 pour constituer le "nouveau fonds » des planches paléographiques de l'établissement ${ }^{8}$.

La Première Guerre mondiale, qui fit disparaittre plusieurs de ces dessins comme celui de Notre-Dame de l'Épine9 , détruit dans les bombardements de Reims, ouvrit une parenthèse scientifique d'un demi-siècle en France. Comme pour d'autres domaines, ce sont les allemands et les Anglo-saxons qui veillèrent à entretenir le flambeau. Le Carnet de Villard fut réédité par Hans Hahnloser à Vienne en 1935, dans un contexte si peu propice que les Français ne découvrirent cette entreprise qu'au moment de sa réimpression en 1972. Aux USA, de fins connaisseurs de l'architecture gothique restaient à l'affut d'opportunités d'acquisition, comme Robert Branner qui a milité pour l'entrée au Cloisters museum, en 1968, d'un dessin de portail oublié des Français depuis sa publication à Montpellier en 1850 (ill. 3) ${ }^{10}$.

La relance des études au cours des années 1980 a donc constitué un sursaut salutaire, orchestré en France par Roland Recht ${ }^{11}$. Il serait vain d'en résumer ici toutes les avancées. Retenons que la place de ces dessins dans les expositions est désormais légitimée au-delà de l'histoire de l'architecture, comme on l'a récemment vu au Grand-Palais en 2010 (1500 : L'Art en France entre Moyen-Âge et Renaissance), aux Archives nationales en 2012 (La Demeure médiévale à Paris) et au Palais des Beaux-Arts de Lille en 2013 (Illuminations) ; et que les découvertes bénéficient désormais d'une plus large publicité. Mais aujourd'hui comme hier, ce sont les trouvailles fortuites qui enrichissent régulièrement le corpus et, dans la majorité des cas, il s'agit de la découverte de remplois de dessins sur parchemin en couvertures de registres ${ }^{12}$.

\section{Quelques destins singuliers}

La plupart des dessins isolés ont eu, en effet, un destin capricieux voire rocambolesque. Mais quelques-uns, on va le voir, ont été de longue date l'objet d'une attention particulière. Le projet dessiné pouvant être, par nature, le témoin d'une réalisation jamais menée à son terme ni même entreprise, ou disparue sans avoir

8. Emmanuel Poulle, "Les fac-similés ", dans L'École nationale des chartes : histoire de l'école depuis 1821, Paris-Thionville, 1997, p. 39-49. Dans le recueil de 1878, le dessin est identifié à un "portail d'église "; il s'agit en réalité de la porte d'un passage couvert.

9. Voir la récente mise au point de Jean-Pierre Ravaux à son sujet dans «Recueil de textes concernant les origines, la construction et le mobilier de Notre-Dame de l'Épine ", Études marnaises, t. CXXII, 2007, p. 337-339.

10. Robert Branner, "A Fifteenth-century French Architectural Drawing at the Cloisters ", Metropolitan Museum Journal, 1976, vol. 11, p. 133-136.

11. Les Bâtisseurs des cathédrales gothiques (catalogue de l'exposition, Strasbourg, 3 septembre-26 novembre 1989), sous la dir. de Roland Recht, Strasbourg, Musées de la ville, 1989. Roland Recht, Le Dessin d'architecture. Origine et fonction, Paris, Adam Biro, 1995.

12. Laurent Vissière, "Un plan de château français du début du $\mathrm{XVI}^{\mathrm{e}}$ siècle ", Bulletin monumental, 2004, p. 197-202 ; Dany Sandron, "Un dessin d'architecture du XV $\mathrm{XV}^{\mathrm{e}}$ siècle pour la loge de mer de Perpignan ", Revue de l'art. Architectures 1400, no 166, 2009-4, p. 91-96. 
laissé de trace, il a souvent alimenté les spéculations en autorisant la restitution d'états disparus ou projetés. Dans certains contextes, il a même retrouvé une finalité pratique en devenant le support de créations historicisantes.

Il en va ainsi de la réutilisation, longtemps après leur création, de dessins pour terminer des édifices gothiques laissés inachevés. Les exemples amplement commentés des façades des cathédrales de Cologne et de Milan au XIX ${ }^{\mathrm{e}}$ siècle viennent immédiatement à l'esprit. On le sait moins, cette démarche a connu un certain engouement en France. On peut douter qu'elle ait été envisagée par Viollet-le-Duc au moment de terminer la cathédrale de Clermont-Ferrand vers 1855-1864 dans la mesure où l'architecte ne semble pas avoir eu connaissance du projet dessiné de façade tracé vers 1496. Au demeurant, ce dessin partiel n'aurait guère été aisément exploitable ${ }^{13}$. Elle a en revanche été engagée à la cathédrale de Beauvais pour laquelle plusieurs versions redessinées d'un prétendu dessin ancien furent publiées en 1824 et 1838 à l'appui de projets de construction d'une façade occidentale (ill. 4) ${ }^{14}$. L'existence même de ce dessin reste cependant sujette à caution. Et l'on ne peut exclure qu'un faux médiéval ait alors été produit pour justifier des projets d'achèvement aux enjeux importants, qu'ils soient politiques ou archéologiques. Mais la fin justifiait les moyens et, au début du, XIX ${ }^{\mathrm{e}}$ siècle, l'idée que l'on pût achever une cathédrale sur un plan apocryphe n'était pas aussi choquante qu'elle l'est devenue depuis la Charte de Venise.

C'est ce qui aurait pu advenir à Saint-Ouen de Rouen sans les aléas de l'histoire et les ambitions des restaurateurs modernes. L'impact des superbes gravures publiées par dom Pommeraye au XVII ${ }^{\mathrm{e}}$ siècle dans la fortune historique de ce monument laissé inachevé a été parfaitement mis en lumière. La légende qui accompagne la planche montrant l'élévation de la façade gothique complète laisse entendre que l'auteur l'a fait graver d'après un dessin d'architecte ${ }^{15}$. L'existence de ce type de projet semble accréditée par la légende d'un dessin à la plume acquis dans le commerce en 1851 et déposé à la bibliothèque municipale de Rouen (ill. 5). Daniel Ramée, qui le fit alors graver, pensait qu'il s'agissait d'une copie du début du XVII ${ }^{e}$ siècle d'une variante du projet reproduit par Pommeraye ${ }^{16}$. Reste à savoir si ces originaux fantomatiques dataient, comme on le pensait au XIX ${ }^{\mathrm{e}}$ siècle, des années 1490-1520, époque de la mise en chantier de la façade, ou du XVII ${ }^{e}$ siècle comme le supposait récemment Jacques Henriet $^{17}$, ce qui en ferait un témoignage passionnant, mais

13. Michael T. Davis, " "Troys Portaulx et Deux Grosses Tours" : The Flamboyant Façade Project for the Cathedral of Clermont ", Gesta, vol. 22, no 1, 1983, p. 67-83; et en dernier lieu ma notice dans 1500 : l'art en France entre Moyen Âge et Renaissance, Paris, 2010, cat. $\mathrm{n}^{\circ} 1$.

14. Florian Meunier, La Cathédrale Saint-Pierre de Beauvais : histoire, architecture, décoration, t. III, le transept, Beauvais, 2006, p. 20-21. Ou Martin et Pierre Chambiges. Architectes des cathédrales flamboyantes, Paris, Picard, 2015, p. 147-148.

15. Cette planche fut reprise en 1655 dans la Topographie de Claude Chastillon.

16. Daniel Ramée, L'Art et l'archéologie au XIX eiècle. Achèvement de Saint-Ouen de Rouen, Paris, Victor Didron, 1851, p. 17-25.

17. Notice sur l'ouvrage de Pommeraye dans Le Gothique retrouvé avant Viollet-le-Duc, Paris, 1979, cat. 116 , p. 66. 


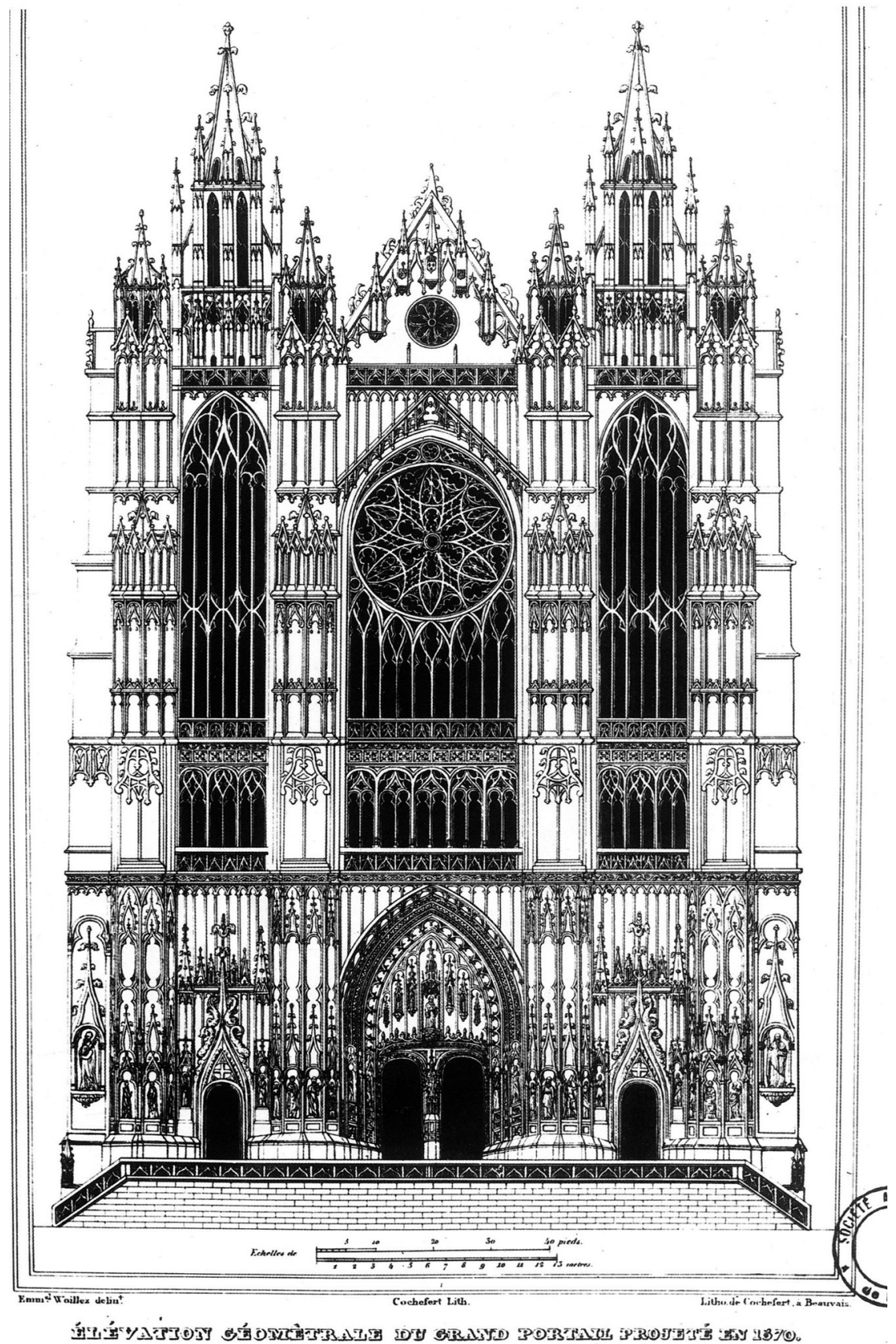

Ill. 4 : Élévation géométrale du grand portail projeté en 1570, gravure publiée par Emmanuel Woillez en 1838. D'après F. Meunier, La Cathédrale Saint-Pierre... op. cit. 


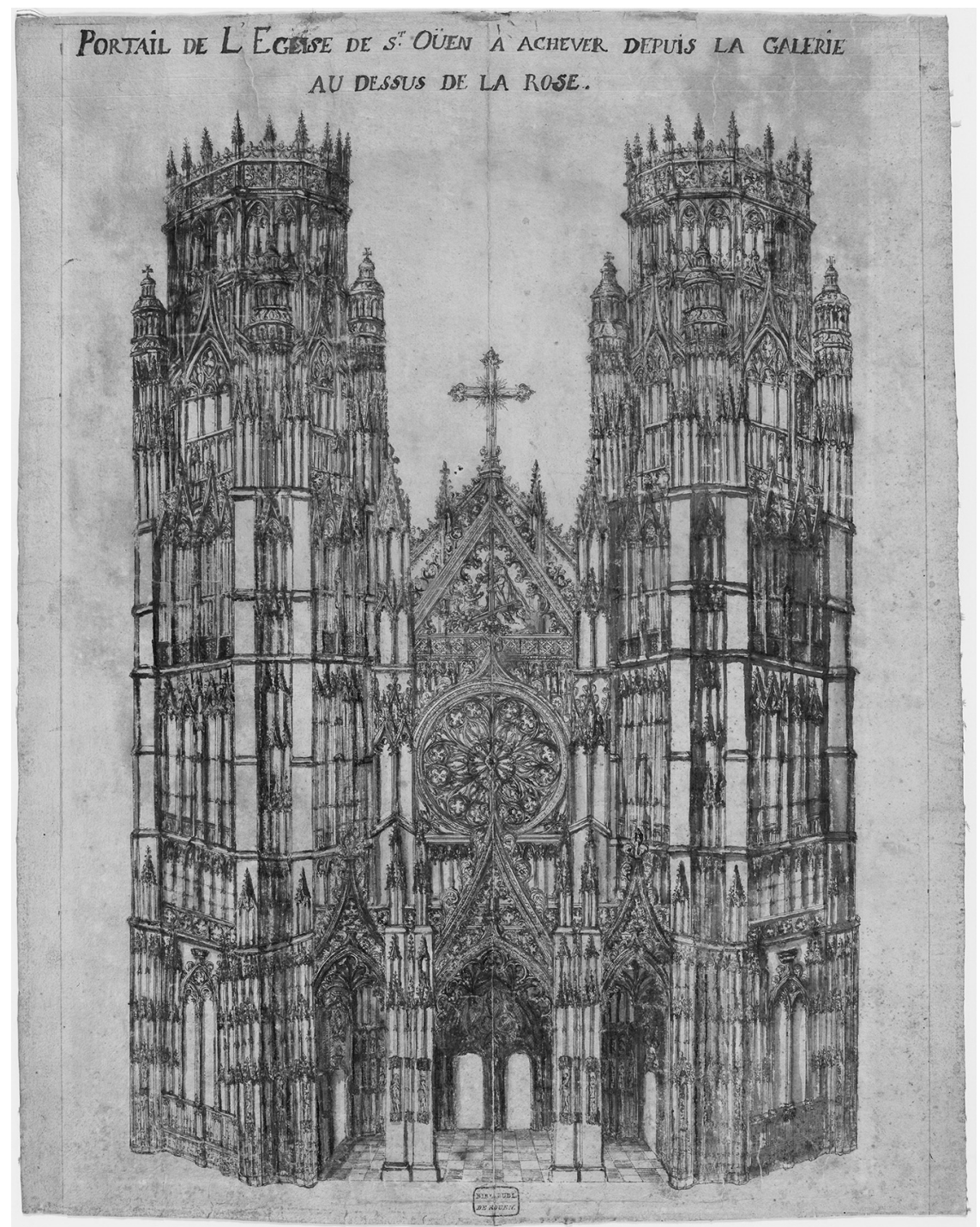

Ill. 5 : Portail de l'église de Saint-Ouen à achever depuis la galerie au-dessus de la rose, dessin à la plume sur papier, $54 \times 38 \mathrm{~cm}$. Rouen, Bibl. mun. Est. topo. g 5518. (c) Rouen nouvelles bibliothèques.

pas unique, du gothic survival de l'époque classique. De cette nature, en effet, semble être le dessin du fonds Robert de Cotte pour la flèche de la Sainte-Chapelle ${ }^{18}$. On connaît la suite du dossier rouennais grâce aux vives controverses soulevées par le

18. BnF, Est., Va 441 (fonds Robert de Cotte, $\mathrm{n}^{\circ} 2580$ ). 
choix du programme définitif de Grégoire en $1845^{19}$ : l'architecte renonça à ses premiers projets d'achèvement respectueux du dessin de Pommeraye et arasa l'ébauche de façade à tour biaises laissée par les architectes de l'époque flamboyante pour bâtir une composition régulière dans le goût du XIV ${ }^{\mathrm{e}}$ siècle. Il faut dire que les membres de la commission des Monuments historiques étaient fort mal disposés vis-à-vis de ce témoignage des fantaisies du gothique tardif : l'un d'eux ne déclarait-il pas que "les amorces de tours déjà construites sont d'une mauvaise époque et feraient un détestable effet si elles étaient achevées ${ }^{20}$."

La possibilité pour les dessins d'architecture de circuler comme modèles à travers l'espace et le temps a scellé le destin d'un autre dessin gothique rouennais moins connu : un projet pour la flèche de l'église Saint-André, édifice construit dans les années 1540. Ce dessin est signalé "à la Vaticane " au début du XVIII siècle par Du Souillet, l'éditeur de l'Histoire de Rouen de Farin (éd. de 1731, t. II, p. 110). En 1860, ce dessin fut redécouvert à la bibliothèque des Augustins de Rome (Angelica) par le peintre et archéologue André Durand alors qu'il dessinait les monuments de Rome pour la collection Demidoff. L'artiste s'en procura une copie qui fut gravée en 1862 par Eustache de la Querrière ${ }^{21}$ (ill. 6). Aux dires de ce dernier, le dessin était annoté d'une écriture italienne "de la fin du XVI siècle" avec la formule "Piante del campanile della chiesa di san andrea alla cita di rouen, anno $1588 \%$. Une tradition locale des plus fragiles veut que le pape lui-même se soit fait envoyer ce relevé de flèche gothique ${ }^{22}$ ! Le document italien, étant désormais introuvable, le dossier reste pour l'heure en suspens ${ }^{23}$.

En plus des fantômes de Saint-Ouen et de Saint-André, Rouen abrite un authentique revenant qui se trouve être le dessin technique français le plus abouti par la qualité du trait et l'association d'un plan et d'une vue géométrale : le projet de "flèche" du trésor de la cathédrale de Rouen ${ }^{24}$. Ce très long parchemin aurait été découvert par un bibliothécaire rouennais chez un bouquiniste du Havre, qui l'avait acquis d'un marchand de ferraille qui le tenait lui-même d'un ex-gardien de musée de Paris! Ou comment brouiller les pistes pour dissimuler une origine sans doute plus proche mais plus douteuse! C'est le chapitre de Rouen, composé d'érudits

19. Jean-Michel Leniaud, Fallait-il achever Saint-Ouen de Rouen? Débats et polémiques. 1837-1852, Rouen, 2002.

20. Françoise Bercé, Les Premiers travaux de la Commission des monuments historiques, 1837-1848: procès-verbaux et relevés d'architectes, Paris, 1979, p. 139.

21. Eustache de La Querrière, Saint-André-de-la-Ville, église paroissiale de Rouen, supprimée en 1791, Rouen, 1862.

22. Elle est rapportée par François Verdier dans «Le beurre et la couronne ", In situ, 1, 2001, note 4 ; URL : http://insitu.revues.org/1148, consulté le 2 février 2015.

23. Je remercie Mathieu Deldicque pour ses patientes investigations dans les bibliothèques romaines à la recherche de ce dessin.

24. Le Trésor de la cathédrale de Rouen, cat. d'expo. du Musée des Antiquités de la Seine-Maritime, 1993, p. 99-100. De nouvelles pistes sur l'origine de ce dessin sont ouvertes dans Antoinette et Jacques Sangouard, "Les sources normandes dans l'achèvement de la cathédrale Sainte-Cécile d'Albi à la fin du Moyen Âge", Bulletin monumental, t. 169-4, 2011, p. 319-334.

Livraisons J'bistoire de l'architecture $n^{\circ} 30$ 


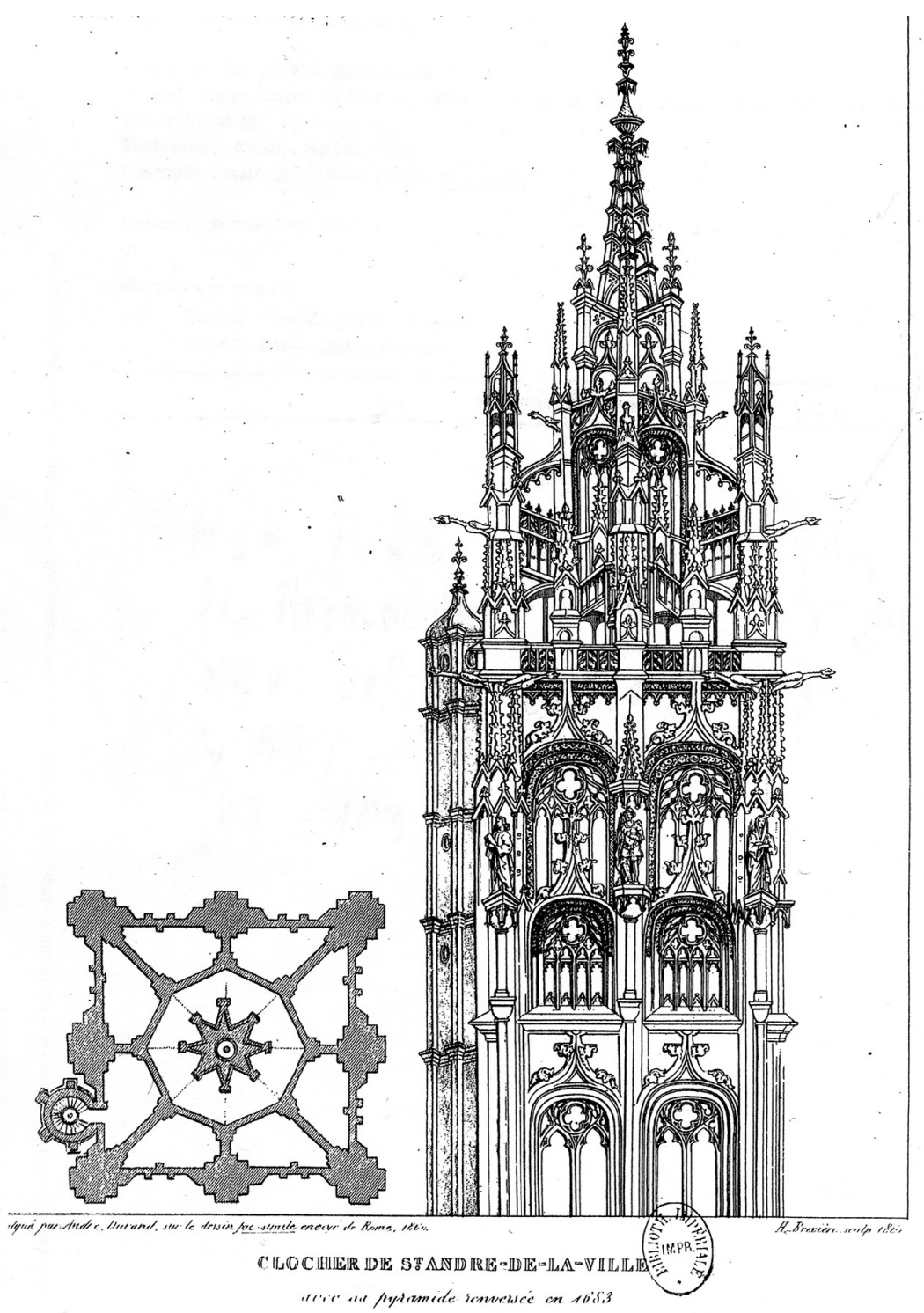

Ill. 6 : Projet (plan et élévation) pour la flèche de l'église Saint-André de Rouen (v. 1540) à la Bibliotheca Angelica à Rome. Gravure dans E. de la Querrière, Saint-André-de-la-Ville..., op. cit.

chanoines, qui s'en porta acquéreur en 1887 ; et c'est l'archiviste de la communauté, l'abbé Sauvage, qui en assura la publication. Sa nature exacte reste malheureusement impossible à établir puisque le dessin est aujourd'hui incommunicable faute d'appartenir à un fonds doté de moyens de conservation et de communication dont devrait pourtant bénéficier ce type de document tout à fait exceptionnel en France. 


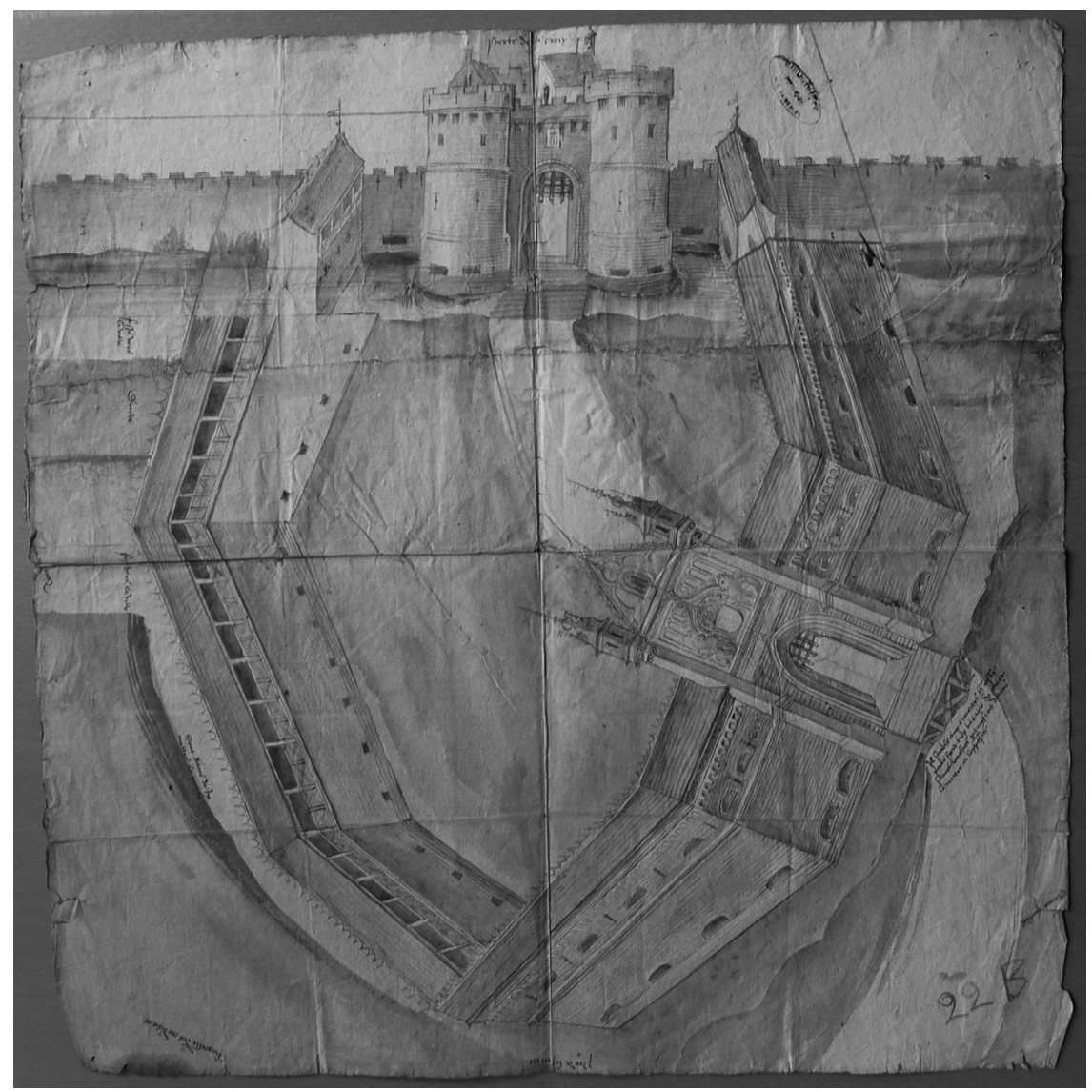

Ill. 7 : Projet de boulevard au-devant de la porte Sainte-Croix de l'enceinte urbaine de Saint-Omer, encre et lavis sur papier (v. 1500). $56 \times 56,5 \mathrm{~cm}$, Saint-Omer, BASO, "cumulus ", anc. Coté 22 B. (c) Cliché Étienne Hamon.

En somme, le panorama esquissé ici fait apparaître une situation très contrastée quant à la qualité et à la finalité de ces dessins d'architecture gothique français et quant à leur sort passé ou présent. Il semble pourtant plus conforme à la réalité de la pratique médiévale et de la fortune moderne de ces dessins que l'image qu'en renvoient les vastes collections germaniques et leurs chefs d'œuvre de géométrie spatiale. Les dessins allemands, souvent privilégiés dans la démonstration du rôle du dessin dans la chaine opératoire de l'architecture, témoignent pour l'essentiel, en effet, d'un aspect très particulier de ce processus : la recherche d'excellence technique et de théorisation dans des milieux savants à la fin de l'époque gothique. Cette démarche a pu exister à la marge en France, pays marqué par un plus grand individualisme des architectes et par une moindre sélection par la théorie dans l'accès aux métiers du bâtiment. Les pièces issues du milieu français restant à découvrir, à 
identifier ou à retrouver çà et là ont peu de chance d'être aussi élaborées que celles d'outre-Rhin. L'enquête systématique n'en est pas moins nécessaire pour saisir la place exacte du dessin dans le processus de conception et de consommation de l'architecture gothique sur ses terres d'origine. En attendant, notre horizon s'élargit de jour en jour grâce aux redécouvertes facilitées par les campagnes de numérisation des fonds patrimoniaux. En 2014, ont ainsi été mis en ligne plusieurs dessins méconnus de monuments de la ville de Saint-Omer (ill. 7). Une série de quelques pièces qui pourrait valoir à la bibliothèque de l'agglomération de cette ville le titre de dépôt français le plus riche en dessins d'architecture gothique, si l'on excepte Strasbourg.

Étienne HAMON

Université de Picardie-Jules Verne | équipe TrAme ${ }^{25}$

25. Je remercie pour ses précieux conseils Yves Gallet et pour leur contribution aux recherches bibliographiques les étudiants de Master de l'Université d'Amiens. 
